\title{
Unlocking vendor-specific tags: Three-dimensional printing of echocardiographic data sets
}

\author{
Ahmed Hosny, MS, ${ }^{a}$ Tao Shen, MBBS, ${ }^{b}$ Alexander S. Kuo, MD, ${ }^{b}$ Dustin Long, MD, \\ Michael N. Andrawes, MD, ${ }^{\mathrm{b}}$ and Joshua D. Dilley, MD, ${ }^{\mathrm{b}}$ Boston, Mass
}

From the "Computational Imaging and Bioinformatics Laboratory, Department of Radiation Oncology, Dana-Farber Cancer Institute, and the ${ }^{\mathrm{b}}$ Department of Anesthesia, Critical Care, and Pain Medicine, Massachusetts General Hospital, Harvard Medical School, Boston, Mass.

Disclosures: Authors have nothing to disclose with regard to commercial support. The Web site and online resources will remain as freeware. The authors have not and will not make any financial gains through the use of materials presented in this article.

Received for publication June 7, 2017; revisions received Aug 11, 2017; accepted for publication Aug 23, 2017; available ahead of print Sept 21, 2017.

Address for reprints: Joshua D. Dilley, MD, Department of Anesthesia, Critical Care and Pain Medicine, Massachusetts General Hospital, 55 Fruit St, Boston, MA 02114 (E-mail: joshuaddilley@gmail.com).

J Thorac Cardiovasc Surg 2018;155:143-5

$0022-5223 / \$ 36.00$

Copyright (c) 2017 by The American Association for Thoracic Surgery

http://dx.doi.org/10.1016/j.jtcvs.2017.08.064

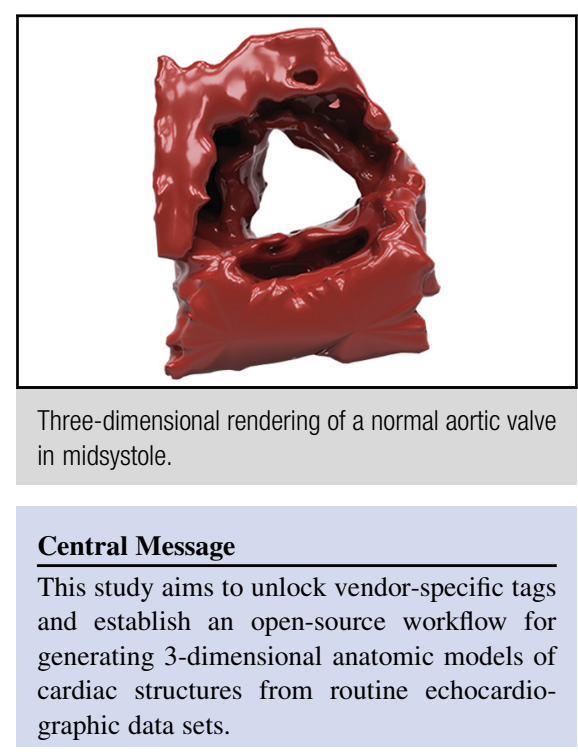

Supplemental material is available online.

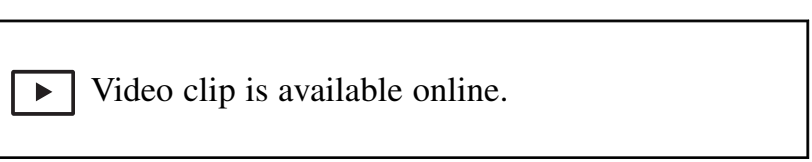

See Editorial Commentary page 146.

Interest in 3-dimensional (3D) printing of anatomic structures continues to grow for a range of applications within the medical field. Proprietary software limits the accessibility of information stored within echocardiographic data sets. This study aims to unlock vendor-specific tags and establish an open-source workflow for generating 3D anatomic models of cardiac structures from routine clinical echocardiographic data sets.

\section{CHALLENGES IN 3D PRINTING OF \\ ECHOCARDIOGRAPHIC DATA SETS}

The use of 3D prints has been reported in many areas of health care, including planning of percutaneous cardiovascular procedures, congenital heart surgery, and medical education. ${ }^{1}$ Projects currently underway at our institution use the prints for sizing neochords used in mitral valve repair and for sizing and placing left atrial appendage occlusion devices.

Previous work with echocardiographic data sets has relied on proprietary software and 3D transesophageal echocardiographic data sets to $3 \mathrm{D}$ print the mitral valve annulus and leaflets. ${ }^{2}$ The software requires user interpretation of the echocardiographic images with manual overlay and assignment of data points to create the 3D model, which is then printed. This technique can lead to loss of anatomic detail and preclude the ability to print the leaflet and annular thicknesses accurately from actual echocardiographic data. Current 3D transesophageal echocardiographic modeling is often limited to restrictive proprietary software packages that focus on the mitral valve alone. Because of these limitations, data sets are difficult to process.

\section{Open-Source Workflow}

Existing 3D transesophageal echocardiographic data sets acquired on Philips IE33 with xMatrix Ultrasound system (Philips Healthcare, Andover, Mass) were exported from Philips QLab (Philips Healthcare) to a 4-dimensional ultrasound DICOM (Digital Imaging and Communications in Medicine) format. The frame of interest is first identified on QLab. For the purposes of our prints, we chose midsystole. The frame was then converted into a NRRD file (the ndimensional Nearly Raw Raster Data format) with custom software developed within this study. NRRD is a widely used imaging file format in scientific visualization and image processing. This NRRD file is then viewed with the free, open source software 3D Slicer (http://www.slicer.org). ${ }^{3}$ Threshold setting of the gray-scale data set was performed in 3D Slicer to generate the desired surface, which was then exported to standard stereolithography STL file format. The STL file was further cropped to the region of interest with Meshmixer, another free software package (http://www. meshmixer.com). 


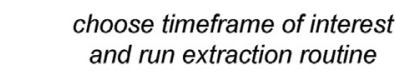

4D ultrasound volume in single proprietary dicom file 3d ultrasound volume @ timeframe of interest import

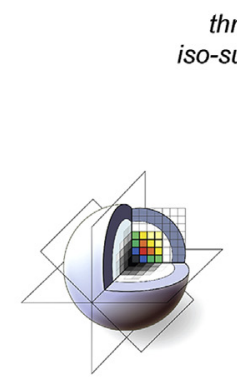

3D Slicer thresholding and

iso-surface generation

FIGURE 1. Graphic representation of process of converting DICOM (Digital Imaging and Communications in Medicine) data sets into 3-dimensional (3d) prints. 4D, 4-Dimensional.

No change to volumetric data occurs during the process of converting ultrasound images to NRRD files. Threshold setting does introduce a source of potential deviation from the original image; however, this deviation is generally negligible. The aortic and mitral valve prints were manually measured and compared with the QLab measurements, with good correlation. To demonstrate general interoperability, the STL files were used to create 3D physical models on a commercially available Form 2 Desktop SLA 3D printer (Formlabs, Inc, Somerville, Mass). Figure 1 illustrates the entire workflow.

The custom software developed runs entirely within the browser and requires no downloads. It is accompanied by a step-by-step instructional video (Video 1) of the entire process (see Appendix). We successfully generated multiple prints from 3D echocardiographic data sets of various cardiac structures (Figure 2). Time for data processing was less than 5 minutes. The time required by the $3 \mathrm{D}$ printer was the predominant limiting factor, and this and varies with specific printer settings.

\section{Unlocking Vendor-Specific Tags}

One of the main challenges facing the use of 3D echocardiographic data in research is the lack of a standardized format for 3D ultrasound data storage. There is almost no interoperability among vendors and standalone software

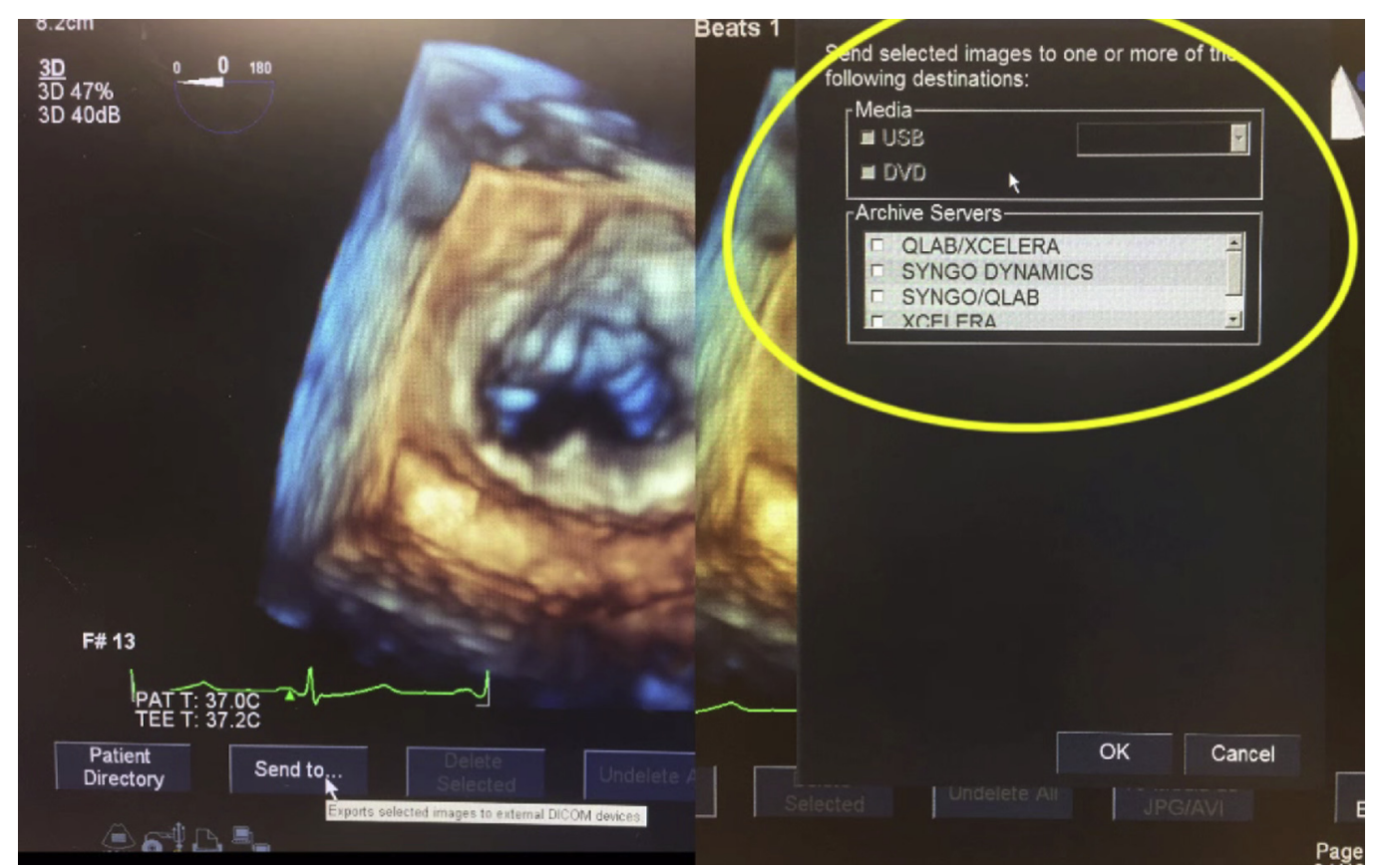

VIDEO 1. Video walk-through illustrating the workflow, from extracting the DICOM (Digital Imaging and Communications in Medicine) files from the Philips IE33 echocardiographic machine (Philips Healthcare, Andover, Mass) to generating the stl file in 3D Slicer. (Available online at https://youtu.be/ LQLC31QJaW.) Video available at: http://www.jtcvsonline.org/article/S0022-5223(17)31826-3/fulltext. 

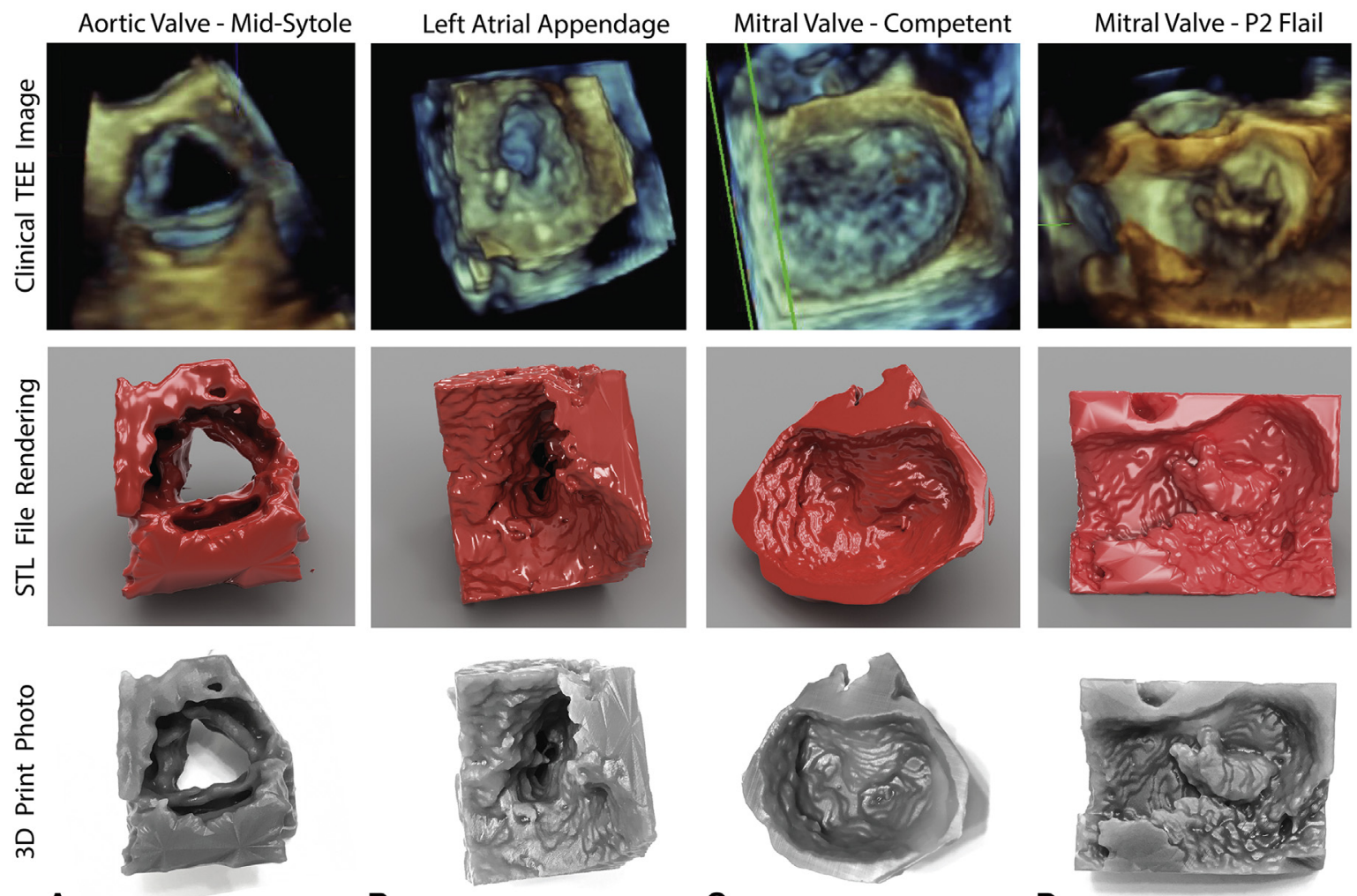

A

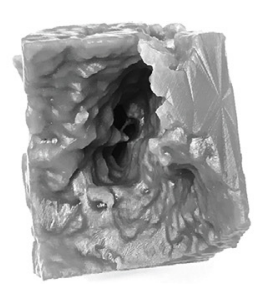

B

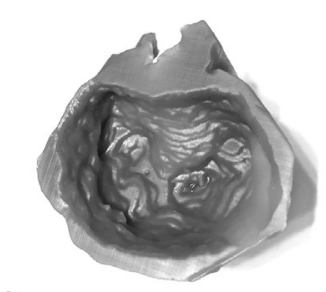

C

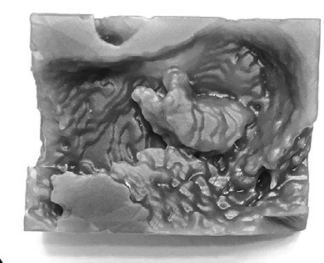

D

FIGURE 2. Side-by-side comparisons of original clinical transesophageal echocardiographic (TEE) images (top), computer-generated renderings of the 3dimensional $(3 D)$ stereolithographic ( $S T L)$ mesh file as produced by the described workflow (middle), and photos of 3D prints produced by a desktop 3D printer (bottom) for 4 valves in 3 routinely acquired views. $A$, A normal aortic valve in midsystole; $B$, a normal left atrial appendage; $C$, a normal mitral valve; $D$, a mitral valve with a posterior leaflet segment $(P 2)$ flail.

packages. Many essential parameters are stored in vendorspecific "private tags" (see Appendix). Although the community attempts to exert significant pressure on vendors to cooperate in developing a DICOM standard for 3D ultrasound imaging data, the export of such data remains a problem. ${ }^{4}$ Our method bypasses interoperability issues until standards are set, with certain limitations (see Appendix). We also use the free open-source 3D Slicer, which unlike a typical radiology workstation is not tied to specific hardware and provides versatile visualizations and advanced functionality.

The quality of prints of a specific structure depends on the choice of modality best suited for that structure. Studies of 3D prints from computed tomographic and magnetic resonance imaging scans have been abundantly reported in the literature, because there is better standardization of data from the DICOM data sets obtained from these scanners than of those from ultrasound. Although 3D echocardiography is not the best imaging choice for every cardiac structure, echocardiographic data sets are very commonly obtained for cardiac surgical patients. Our technique provides the ability to create nearly exact prints of 3D images generated by the ultrasound machine. If the image does not accurately represent particular elements of a structure, however, neither will the print. Use of the free tool that we have developed to unlock the ultrasound data sets will allow for the same ease in processing as computed tomographic and magnetic resonance imaging data sets. Our method is free, open, and efficient. It is our hope that this technique will further enable clinicians and researchers to generate 3D prints from 3D ultrasound data sets.

\section{References}

1. Costello JP, Olivieri LJ, Su L, Krieger A, Alfares F, Thabit O, et al. Incorporating three-dimensional printing into a simulation-based congenital heart disease and critical care training curriculum for resident physicians. Congenit Heart Dis. 2015;10:185-90.

2. Mahmood F, Owais K, Taylor C, Montealegre-Gallegos M, Manning W, Matyal R, et al. Three-dimensional printing of mitral valve using echocardiographic data. JACC Cardiovasc Imaging. 2015;8:227-9.

3. Fedorov A, Beichel R, Kalpathy-Cramer J, Finet J, Fillion-Robin JC, Pujol S, et al 3d Slicer as an image computing platform for the Quantitative Imaging Network. Magn Reson Imaging. 2012;30:1323-41.

4. Preim B, Botha CP. Visual computing for medicine: Theory, algorithms, and applications (the Morgan Kaufmann series in computer graphics). 2nd ed. Philadelphia: Elsevier Science; 2013:55 


\section{APPENDIX. SUPPLEMENTAL MATERIAL Open-Source Tool and Tutorial}

A Web-based tool has been developed to extract ultrasound slices at time frame of interest from 3-dimensional (3D) ultrasound DICOM (Digital Imaging and Communications in Medicine) files. The tool was developed to run in the browser as a client-side-only application and therefore developed in JavaScript. This ensures confidentiality because patient data are not processed on remote servers. The code is made available as an open-source code repository at https://github.com/ ahmedhosny/ultrasound-converter, and the Web application can be found at https://ahmedhosny.github.io/ultrasoundconverter/ (Chrome browser support only). In addition, we have included on the Web site a video walk-through illustrating the workflow, from extracting the DICOM files from the Philips IE33 echocardiographic machine (Philips Healthcare) to generating the .stl file in 3D Slicer. The video is available directly at https://youtu.be/LQLC31QJaWI. Current work is underway to expand supported files beyond those exported by Philips QLab software (Philips Healthcare), expand export file types such as DICOM, and expand supported browsers.

\section{Example of Vendor-Specific Tag}

A 3D volume in a single DICOM file exported from a Philips QLab workstation shows slice spacing (or pixel Z height, essential for the $3 \mathrm{D}$ reconstruction of the data set) in a tag labeled "(3001, 1003) PrivateTagData." The DICOM standards specify that slice spacing should live in the "(0018, 0050) SliceThickness" tag. This 3D ultrasound multiframe DICOM file is therefore only correctly parsed by the software that exported it, and any attempt to parse it with other software is bound to return inaccurate results or be incompletely unsuccessful.

\section{Limitations}

Although our method currently only deals with files generated from Philips QLab, it is fully extensible to other workstation models. One limitation arises with this proposed method: the loss of patient identifiers and information when converting DICOMs into other file formats. This can be remedied by dumping the extracted information from vendor DICOM files into entirely standard DICOM files. 